\title{
ORIGINAL
}

\section{Source-specific host response and outcomes in critically ill patients with sepsis: a prospective cohort study}

\author{
Hessel Peters-Sengers ${ }^{1,2^{*}}$ (D) Joe M. Butler ${ }^{1,2}$ (D) Fabrice Uhel ${ }^{1,2}$, Marcus J. Schultz ${ }^{3,4,5,6}$, Marc J. Bonten ${ }^{7,8}$, \\ Olaf L. Cremer ${ }^{9}$, Brendon P. Scicluna ${ }^{1,2,10}$, Lonneke A. van Vught ${ }^{1,2}$ and Tom van der Poll ${ }^{1,2,11}$ on behalf of the \\ MARS consortium
}

(c) 2021 The Author(s)

\begin{abstract}
Purpose: There is limited knowledge on how the source of infection impacts the host response to sepsis. We aimed to compare the host response in sepsis patients with a single, known source at admission $(<24 \mathrm{~h})$ to the intensive care unit.

Methods: From the molecular diagnosis and risk stratification of sepsis (MARS) prospective cohort, we measured 16 plasma host response biomarkers reflective of key host response pathways in 621 sepsis patients. In a subgroup ( $n=335)$, blood leukocyte transcriptomes were compared between the sources. Differences in clinical patient profiles and survival were compared in the whole sepsis cohort $(n=2019)$.

Results: The plasma biomarker cohort was categorized into sepsis originating from the respiratory tract $(n=334,53.8 \%)$, abdomen $(n=159,25.6 \%)$, urinary tract $(n=44,7.1 \%)$, cardiovascular $(n=41,6.6 \%)$, central nervous system (CNS) $(n=18$, $2.9 \%)$, or skin $(n=25,4 \%)$. This analysis revealed stronger inflammatory and cytokine responses, loss of vascular integrity and coagulation activation in abdominal sepsis relative to respiratory. Endothelial cell activation was prominent in urinary, cardiovascular and skin infections, while CNS infection was associated with the least host response aberrations. The leukocyte transcriptional response showed the largest overlap between abdominal and pulmonary infections (76\% in common); notable differences between the sources were detected regarding hemostasis, cytokine signaling, innate and adaptive immune, and metabolic transcriptional pathways. After adjustment for confounders, the source of infection remained an independent contributor to 30-day mortality (unadjusted $p=0.001$, adjusted $p=0.028$ ).
\end{abstract}

Conclusion: Sepsis heterogeneity is partly explained by source-specific host response dysregulations and should be considered when selecting patients for trials testing immune modulatory drugs.

Keywords: Sepsis, Source of infection, Site of infection, Host response, Intensive care unit

\footnotetext{
*Correspondence: h.peterssengers@amsterdamumc.nl

${ }^{1}$ Center for Experimental and Molecular Medicine, Amsterdam University

Medical Centers, Location Academic Medical Center, University

of Amsterdam, Room T1.240, Meibergdreef 9, 1105 AZ Amsterdam, The

Netherlands

Full author information is available at the end of the article

Hessel Peters-Sengers and Joe M. Butler share the first authorship.
}

The members of the MARS consortium are listed in the Acknowledgements.

\section{实 Springer}




\section{Introduction}

The incidence of sepsis, defined as life-threatening organ dysfunction due to a dysregulated host response to infection [1], has been estimated at 49 million cases associated with 11 million sepsis-related deaths in 2017 [2]. To develop urgently required treatments, strategies are needed to better understand the heterogeneity in the host response [3, 4]. The trigger of the dysregulated host response is an uncontrolled infection arising from a specific anatomic source. Knowledge on differences in the host response during sepsis originating from different sources is limited.

Most research on the source-specific peculiarities of sepsis has focused on clinical patient data [5-11]. These studies showed significant source-specific differences with regard to patient characteristics, organs affected, cultured pathogens, supportive treatments, and length of hospital stay. Differences in natural killer cell, lymphocyte and monocyte counts and apoptosis rates have been observed between sepsis patients with different sources of infection $[12,13]$ as well as different expression of pattern-recognition receptors on neutrophils and monocytes [14]. Another study compared blood leukocyte transcriptomes between patients with sepsis due to community acquired pneumonia or fecal peritonitis, reporting a largely common transcriptomic response with the expression of only a modest number of genes being dependent on the source of infection [15]. A recent study stratified 316 patients admitted to a surgical intensive care unit (ICU) into five groups based on the site of their inciting infection [16]. Sequential plasma interleukin (IL)-6 and IL-8 measurements documented a large overlap between groups, with more sustained elevations in patients with an abdominal source [16].

We hypothesized that the specific source of infection at admission to the ICU, categorized into respiratory, abdominal, urinary, cardiovascular, central nervous system (CNS), and skin infection, is associated with different host response aberrations. To address this hypothesis, we conducted a prospective observational study in two mixed ICUs in the Netherlands during a 3-year period. By measuring plasma biomarkers indicative of organ systems known to be disturbed in sepsis and by applying whole-blood leukocyte transcriptome profiling, we provide an in-depth insight into the dysregulated biologic pathways in sepsis patients stratified according to the source of infection at ICU admission.

\section{Methods}

\section{Study population and design}

This study was part of the Molecular Diagnosis and Risk Stratification of Sepsis (MARS) project, conducted

\section{Take-home message}

The heterogeneity of the host response to sepsis makes stratification of patients into subgroups with more similar pathobiological profiles a major challenge. Our results suggest that the source of infection partly explains sepsis heterogeneity and should be taken into account when selecting patients for trials testing immune modulatory drugs.

between January 2011 and December 2013 in the mixed ICUs of two tertiary teaching hospitals in the Netherlands (Academic Medical Center, Amsterdam and University Medical Center Utrecht, Utrecht; ClinicalTrials.gov identifier NCT01905033) [17-19]. All consecutive patients with sepsis older than 18 years of age were included via an optout consent method approved by the institutional review boards (IRBs) of both hospitals (IRB No. 10-056C). Readmissions within 30 days of ICU discharge and patients transferred from another ICU were excluded, except when patients were referred to one of the study centers the same day of presentation to the first ICU.

\section{Definitions}

For every patient, the plausibility of an infection was assessed daily using a four-point scale (ascending from none, possible, probable, to definite) as described in detail [17]. Sepsis was defined according to the most recent definition; as the presence of an infection with a likelihood of possible, probable or definite) diagnosed within $24 \mathrm{~h}$ after ICU admission and organ dysfunction(s) represented by two or more Sequential Organ Failure Assessment (SOFA) points [1].

The source of infection was determined by trained research physicians by retrospective case-by-case review based on all clinical data, microbiology and radiology results, and the Acute Physiology and Chronic Health Evaluation IV (APACHE IV) primary diagnosis for ICU admission, and further categorized into "pure" sources without coinfection or "mixed" infections (more than one source); sources were respiratory, abdominal, urinary tract, cardiovascular, CNS, skin, unknown source, and other. "Other" consisted of several categories with few patients (eTable 1, electronic supplemental material). Categorization of infections and clinical definitions are described in more detail in the electronic supplemental material (eTable 2; eMethods, respectively).

\section{Biomarker assays}

In the subgroup of patients enrolled during the first 2.5 years with a probable or definite likelihood of infection [17], we analyzed 16 plasma biomarkers reflecting key pathways implicated in sepsis pathogenesis. Urinary tract and skin infections were case-by-case reviewed by 
the research team to assure these were the reason for ICU admission rather than a comorbid condition. For the urinary tract infection group, this resulted in selection of infections with a definite likelihood only [20], whereas in the skin infection group fasciitis necroticans and erysipelas were selected as described [21]. Assays are described in the electronic supplemental material (eMethods).

\section{Whole-blood transcriptomic analyses}

Microarray, RNA processing and bioinformatic analytic strategies are described in the electronic supplemental material (eMethods).

\section{Statistical analyses}

Baseline characteristics were compared with ANOVA for normally distributed and Kruskal-Wallis test for nonnormally distributed continuous variables. Categorical data were analyzed using Fisher's exact test. Crude and adjusted 30-day mortality outcome in different source of infection groups were compared as described in the electronic supplemental material (eMethods), which also includes selection of confounders and handling of missing data. Differences in (log-transformed) plasma biomarker distributions between source of infection groups upon ICU admission were compared using linear regression with contrast dummy coding. As plasma biomarkers are often proportional to disease severity during sepsis [22], results are also adjusted for confounders corresponding to the mortality analyses with a similar imputation approach for missing clinical data as described in the electronic supplemental material (eMethods). Calculation of principal component analysis (PCA) plots was done by a singular value decomposition of the centered and scaled data matrix including the (logged) protein plasma biomarkers for each key immune pathway. Data analyses were performed in R (v3.6.1). Significance level was set to $5 \%$.

\section{Results}

\section{Patient characteristics and outcome}

Of the 8313 ICU admissions enrolled, 6294 were excluded because they did not have sepsis, involved readmissions or were transfers from another ICU (Figure S1, electronic supplementary material). In the remaining 2019 sepsis patients, six single known sources were categorized and clinical characteristics were compared between them (eTable 2; eTable 3, respectively, electronic supplementary material). The mean age was 59.7 years (SD 15.9) and $39.4 \%$ were female. Most patients had a respiratory tract infection $(n=853,42.2 \%)$, followed by abdominal $(n=297,14.7 \%)$, urinary tract $(n=131,6.5 \%)$, skin infection $(n=77,3.8 \%)$, CNS $(n=71,3.5 \%)$, then cardiovascular $(n=68,3.4 \%) ; 74$ patients $(3.7 \%)$ had a relatively rare source of infection, grouped as 'other' (eTable 1 , electronic supplementary material) and 74 patients (3.7\%) had an unknown source. Multiple sources of infection at ICU arrival were found in 373 (18.5\%) patients (eTable 4, electronic supplementary material).

The Charlson comorbidity index score was similar in patients with different sources of infection with the exception of CNS, which was associated with a lower score. The type of chronic comorbidity was heterogeneous between sources, e.g., urosepsis was more frequent among female patients and in those with renal insufficiency or diabetes, while skin sepsis occurred more often in patients with a high BMI and diabetes. On admission to the ICU, shock was more common among patients with abdominal (34.1\%), skin (41.7\%) or mixed infections (34.3\%). Modified SOFA scores were relatively similar between groups apart from CNS infections (relatively low). Blood cultures were most likely to be positive in urosepsis $(37.4 \%)$ whereas they were least likely to be positive in respiratory (11.5\%). Regarding therapy within $24 \mathrm{~h}$ of admission, CNS infections had the highest use of mechanical ventilation and lowest use of vasopressors, demonstrating this source's specific indications for critical care.

Crude mortality at day 30 after admission was significantly higher in respiratory, abdominal, cardiovascular, skin, unknown source, and mixed infections compared to urinary, CNS, and other infections $(p=0.002$; Table 1$)$. Adjustment for factors that could influence survival only modestly altered the incidence of 30-day mortality in different groups, with exception of CNS infection (higher adjusted mortality) due to low disease severity (Fig. 1). Table 2 shows unadjusted and adjusted hazard ratios of 30-day mortality of pairwise comparisons of the six known sources of infection (eTable 5 with inclusion of unknown, other and mixed sources). Mortality up to 1 year was highest in mixed and unknown source, and cardiovascular was highest out of the known sources (Figure S2).

\section{Plasma host response biomarkers}

Biomarkers indicative of host response pathways implicated in sepsis pathogenesis were measured on admission to the ICU in a subgroup of patients enrolled during the first 2.5 years $(n=621,30.8 \%)$. The clinical characteristics of this subgroup were comparable to the whole cohort (Table 1). Figure 2 shows admission biomarker levels in patients with different sources across three pathophysiological domains: systemic inflammation, endothelial cell activation and dysfunction, and coagulation activation. Pairwise unadjusted comparisons between sources are depicted in the electronic supplementary material (eTable 6). 
Table 1 Plasma biomarker cohort: baseline characteristics and outcome of patients with sepsis stratified according to source of infection

\begin{tabular}{|c|c|c|c|c|c|c|c|c|}
\hline & ALL & Respiratory & Abdominal & Urinary & Cardiovascular & CNS & Skin & $p$ value \\
\hline$n$ & 621 & 334 & 159 & 44 & 41 & 18 & 25 & \\
\hline \multicolumn{9}{|l|}{ Demographics } \\
\hline $\begin{array}{l}\text { Age, years, mean } \\
\text { (SD) }\end{array}$ & $60.9(14.7)$ & $60.5(15.5)$ & $62.1(12.6)$ & $61.8(14.8)$ & $61.6(13.1)$ & $58.4(15.1)$ & 56.7 (16.6) & 0.539 \\
\hline $\begin{array}{l}\text { Gender, female } \\
(n, \%)\end{array}$ & $260(41.9)$ & $123(36.8)^{\mathrm{L}}$ & $72(45.3)$ & $29(65.9)^{\mathrm{H}}$ & $14(34.1)$ & $6(33.3)$ & $16(64)$ & 0.001 \\
\hline BMI, mean (SD) & $25.5(6.3)$ & $24.6(5.6)^{\mathrm{L}}$ & $26.1(6.9)$ & $28.4(7.2)^{\mathrm{H}}$ & $25.1(4.5)$ & $25.7(4.1)$ & $28.9(9.6)^{\mathrm{H}}$ & $<0.001$ \\
\hline Race, white, $n$ (\%) & $540(87)$ & $290(86.8)$ & $140(88.1)$ & $38(86.4)$ & $35(85.4)$ & $16(88.9)$ & $21(84)$ & 0.991 \\
\hline Surgical admission, & $160(25.8)$ & $29(8.7)^{\mathrm{L}}$ & $87(54.7)^{\mathrm{H}}$ & $4(9.1)^{\mathrm{L}}$ & $22(53.7)^{\mathrm{H}}$ & $4(22.2)$ & $14(56)^{\mathrm{H}}$ & $<0.001$ \\
\hline
\end{tabular}

Chronic comorbid-

\begin{tabular}{|c|c|c|c|c|c|c|c|c|}
\hline \multicolumn{9}{|l|}{$\begin{array}{l}\text { Chronic comorbid- } \\
\text { ity, } n(\%)\end{array}$} \\
\hline None & $158(25.4)$ & 73 (21.9) & $52(32.7)^{\mathrm{H}}$ & $6(13.6)$ & $11(26.8)$ & $10(55.6)^{\mathrm{H}}$ & $6(24)$ & 0.002 \\
\hline $\begin{array}{l}\text { Cardiovascular insuf- } \\
\text { ficiency }\end{array}$ & $20(3.2)$ & $9(2.7)$ & $6(3.8)$ & $3(6.8)$ & $1(2.4)$ & $0(0)$ & $1(4)$ & 0.699 \\
\hline Renal insufficiency & $89(14.3)$ & $43(12.9)$ & $23(14.5)$ & $17(38.6)^{\mathrm{H}}$ & $2(4.9)$ & $1(5.6)$ & $3(12)$ & $<0.001$ \\
\hline $\begin{array}{l}\text { Respiratory insuf- } \\
\text { ficiency }\end{array}$ & $51(8.2)$ & $44(13.2)$ & $3(1.9)^{\mathrm{L}}$ & $2(4.5)$ & $1(2.4)$ & $0(0)$ & $1(4)$ & $<0.001$ \\
\hline Immune deficiency & $140(22.5)$ & $91(27.2)^{\mathrm{H}}$ & $25(15.7)^{\mathrm{L}}$ & $11(25)$ & $5(12.2)$ & $3(16.7)$ & $5(20)$ & 0.041 \\
\hline Malignancy & $146(23.5)$ & $81(24.3)$ & $48(30.2)^{\mathrm{H}}$ & $6(13.6)$ & $5(12.2)$ & $1(5.6)$ & $5(20)$ & 0.026 \\
\hline COPD & 97 (15.6) & $75(22.5)^{\mathrm{H}}$ & $11(6.9)^{\mathrm{L}}$ & $6(13.6)$ & $4(9.8)$ & $0(0)$ & $1(4)$ & $<0.001$ \\
\hline Diabetes & $131(21.1)$ & 64 (19.2) & $34(21.4)$ & $15(34.1)^{\mathrm{H}}$ & $8(19.5)$ & $1(5.6)$ & $9(36)$ & 0.047 \\
\hline $\begin{array}{l}\text { Charlson score } \\
\text { (median [IQR]) }\end{array}$ & $4[3,6]$ & $4[2,6]$ & $4[3,6]$ & $4.5[3,5.2]$ & $4[3,5]$ & $3[2,4]$ & $4[2,5]$ & 0.376 \\
\hline \multicolumn{9}{|l|}{$\begin{array}{l}\text { Causative patho- } \\
\text { gen }^{A}\end{array}$} \\
\hline $\begin{array}{l}\text { Gram-positive } \\
\text { bacteria }\end{array}$ & $267(43)$ & $105(31.4)^{\mathrm{L}}$ & $91(57.2)^{\mathrm{H}}$ & $8(18.2)^{\mathrm{L}}$ & $32(78)^{\mathrm{H}}$ & $10(55.6)$ & $21(84)^{\mathrm{H}}$ & $<0.001$ \\
\hline $\begin{array}{l}\text { Gram-negative } \\
\text { bacteria }\end{array}$ & $296(47.7)$ & $125(37.4)^{\mathrm{L}}$ & $111(69.8)^{\mathrm{H}}$ & $41(93.2)^{\mathrm{H}}$ & $4(9.8)^{\mathrm{L}}$ & $4(22.2)$ & $11(44)$ & $<0.001$ \\
\hline Fungi & $49(7.9)$ & $32(9.6)$ & $14(8.8)$ & $1(2.3)$ & $1(2.4)$ & $0(0)$ & $1(4)$ & 0.210 \\
\hline Virus & $44(7.1)$ & $41(12.3)^{\mathrm{H}}$ & $2(1.3)^{\mathrm{L}}$ & $1(2.3)$ & $0(0)$ & $0(0)$ & $0(0)$ & $<0.001$ \\
\hline Unknown & $112(18)$ & $80(24)^{\mathrm{H}}$ & $21(13.2)$ & $2(4.5)^{\mathrm{L}}$ & $6(14.6)$ & $3(16.7)$ & $0(0)$ & 0.001 \\
\hline Other & $7(1.1)$ & $2(0.6)$ & $3(1.9)$ & $0(0)$ & $1(2.4)$ & $1(5.6)$ & $0(0)$ & 0.283 \\
\hline $\begin{array}{l}\text { Blood culture posi- } \\
\text { tive (\%) }\end{array}$ & $156(25.1)$ & $48(14.4)^{\mathrm{L}}$ & $53(33.3)^{\mathrm{H}}$ & $21(47.7)^{\mathrm{H}}$ & $13(31.7)$ & $9(50)^{H}$ & $12(48)^{\mathrm{H}}$ & $<0.001$ \\
\hline \multicolumn{9}{|l|}{$\begin{array}{l}\text { Severity of disease } \\
\text { on admission }\end{array}$} \\
\hline $\begin{array}{l}\text { mSOFA score, } \\
\text { median [IQR] }{ }^{\mathrm{B}}\end{array}$ & $7[5,9]$ & $7[4,9]^{\mathrm{L}}$ & $8[6,10]^{\mathrm{H}}$ & $8[5,10]$ & $7[5,8]$ & $3.5[3,5]^{\mathrm{L}}$ & $7[6,9]$ & $<0.001$ \\
\hline $\begin{array}{l}\text { APACHE IV Score, } \\
\text { mean (SD) }\end{array}$ & $82.1(28.8)$ & $82(28.9)$ & $83.1(30.4)$ & $87.4(26.4)$ & $77.5(29.1)$ & $74.7(22.1)$ & $79.6(23.5)$ & 0.530 \\
\hline APS, mean (SD) & $68.9(27.3)$ & $68.1(27.4)$ & $70.8(28.7)$ & $73.7(26)$ & $65.4(27.7)$ & $62.6(18.1)$ & $69.1(25.2)$ & 0.542 \\
\hline $\mathrm{AKI}, n(\%)$ & $161(25.9)$ & $66(19.8)^{\mathrm{L}}$ & $57(35.8)^{\mathrm{H}}$ & $19(43.2)^{\mathrm{H}}$ & $9(22)$ & $1(5.6)$ & $9(36)$ & $<0.001$ \\
\hline ARDS, $n(\%)$ & $139(22.4)$ & $101(30.2)^{\mathrm{H}}$ & $22(13.8)^{\mathrm{L}}$ & $8(18.2)$ & $4(9.8)$ & $1(5.6)$ & $3(12)$ & $<0.001$ \\
\hline Septic shock, n (\%) & $183(29.5)$ & $86(25.7)$ & $68(42.8)^{\mathrm{H}}$ & $9(20.5)$ & $9(22.5)$ & $0(0)^{\mathrm{L}}$ & $11(44)$ & $<0.001$ \\
\hline \multicolumn{9}{|l|}{$\begin{array}{l}\text { Therapy during the } \\
\text { first } 24 \mathrm{~h}\end{array}$} \\
\hline $\begin{array}{l}\text { Mechanical ventila- } \\
\text { tion }(n, \%)\end{array}$ & $509(82)$ & $286(85.6)^{\mathrm{H}}$ & $130(81.8)$ & $25(56.8)^{\mathrm{L}}$ & $32(78)$ & $16(88.9)$ & $20(80)$ & $<0.001$ \\
\hline $\begin{array}{l}\text { Renal replacement } \\
\text { therapy }(n, \%)\end{array}$ & $56(9)$ & $24(7.2)$ & $23(14.5)^{\mathrm{H}}$ & $3(6.8)$ & $1(2.4)$ & $2(11.1)$ & $3(12)$ & 0.078 \\
\hline Vasopressors $(n, \%)$ & $378(60.9)$ & $192(57.5)$ & $113(71.1)^{\mathrm{H}}$ & $24(54.5)$ & $25(61)$ & $3(16.7)^{\mathrm{L}}$ & $21(84)$ & $<0.001$ \\
\hline
\end{tabular}


Table 1 (continued)

\section{Respiratory Abdominal Urinary Cardiovascular CNS}

\section{Outcomes}

\begin{tabular}{|c|c|c|c|c|c|c|c|c|}
\hline $\begin{array}{l}\text { ICU LOS, days, } \\
\text { median [IQR] }\end{array}$ & $4[2,9]$ & $5[2,10]^{\mathrm{H}}$ & $3[1,8.5]$ & $2[1,5]^{\mathrm{L}}$ & $3[2,5]$ & $5[2,8]$ & $3[2,9]$ & $<0.001$ \\
\hline $\begin{array}{l}\text { Hospital LOS, days, } \\
\text { median [IQR] }\end{array}$ & $15[8,32]$ & $14[7,25]^{\mathrm{L}}$ & $19[8,45]^{H}$ & $12.5[7.8,23.2]$ & $26[8,42]$ & $18.5[10.2,41]$ & $20[11,41]$ & 0.022 \\
\hline
\end{tabular}

\section{ICU-acquired com-} plications, $n(\%)^{\mathrm{C}}$

\begin{tabular}{|c|c|c|c|c|c|c|c|c|}
\hline None & $421(67.8)$ & $225(67.4)$ & $102(64.2)$ & $37(84.1)^{\mathrm{H}}$ & $26(63.4)$ & 13 (72.2) & $18(72)$ & 0.215 \\
\hline AKI & $114(18.4)$ & $64(19.2)$ & 28 (17.6) & $3(6.8)$ & $13(31.7)$ & $2(11.1)$ & $4(16)$ & 0.084 \\
\hline ARDS & $59(9.5)$ & $38(11.4)^{\mathrm{H}}$ & $18(11.3)$ & $1(2.3)$ & $1(2.4)$ & $0(0)$ & $1(4)$ & 0.081 \\
\hline
\end{tabular}
tion

Mortality, $\boldsymbol{n}(\%)$

\begin{tabular}{|c|c|c|c|c|c|c|c|c|}
\hline ICU & $116(18.7)$ & $64(19.2)$ & 31 (19.5) & 6 (13.6) & $12(29.3)$ & $0(0)$ & $3(12)$ & 0.119 \\
\hline Hospital & $193(31.1)$ & $104(31.1)$ & $52(32.7)$ & $12(27.3)$ & 15 (36.6) & $5(27.8)$ & $5(20)$ & 0.762 \\
\hline 30 days & $171(27.5)$ & $98(29.3)$ & $46(28.9)$ & $9(20.5)$ & $11(26.8)$ & $2(11.1)$ & $5(20)$ & 0.421 \\
\hline 90 days & $240(38.6)$ & 133 (39.8) & $64(40.3)$ & $13(29.5)$ & $19(46.3)$ & $6(33.3)$ & $5(20)$ & 0.235 \\
\hline 1 year & $306(49.3)$ & $174(52.1)$ & 78 (49.1) & $18(40.9)$ & $22(53.7)$ & 7 (38.9) & $7(28)$ & 0.161 \\
\hline
\end{tabular}

Data presented as median [interquartile range], or $n$ (\%). Continuous variables were compared using the analysis of variance test or Kruskal-Wallis rank-sum test when appropriate, resulting in overall $p$ value. Associations between categorical variables were tested using the Fisher's exact test, resulting in overall $p$ value

CNS central nervous system, APS acute physiology score, ARDS acute respiratory distress syndrome, AKI acute kidney injury, APACHE acute physiology and chronic health evaluation, ICU intensive care unit, SOFA sequential organ failure assessment

$\mathrm{H}$ If significantly higher than the grand mean (mean of means of sources) of the plasma biomarker cohort

$L$ If significantly lower than the grand mean (mean of means of sources) of the plasma biomarker cohort

A In 100 patients (16.1\%) more than one cultured organism was assigned as causative

${ }^{B}$ mSOFA modified sequential organ failure assessment (excluding central nervous system component)

C Complications were defined as ICU-acquired when diagnosed more than $48 \mathrm{~h}$ after admission to the ICU

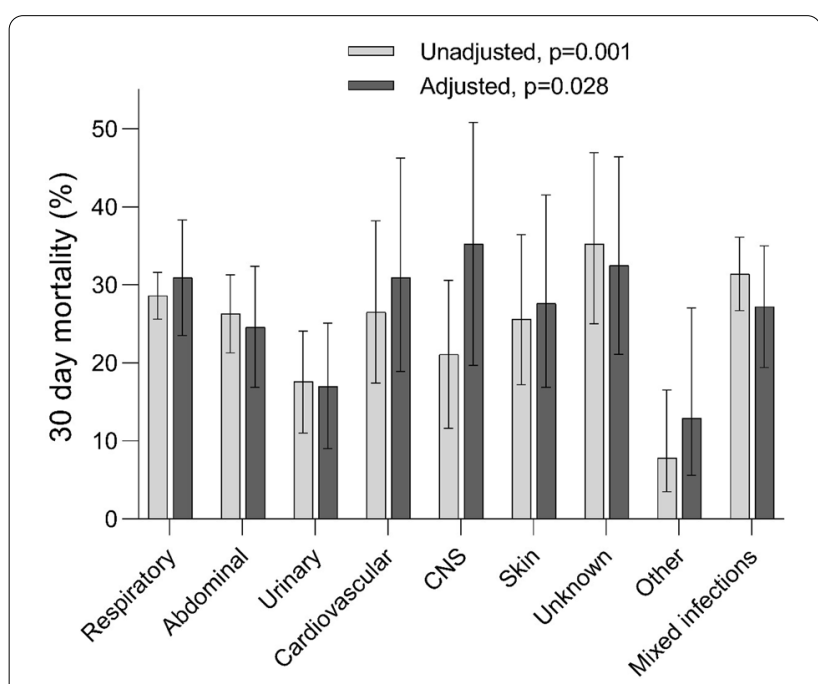

Fig. 1 30-day mortality incidence (\%) among sepsis patients with different sources of infection; $p$ values calculated by type-III overall Wald test. Data are presented as bars with 95\% confidence bands. Adjusted model included age, sex, ethnicity, BMI, Charlson comorbidity score (without age), admission type, hospital site, blood culture positivity, type of causative pathogen, mSOFA score, Acute Physiology Score and shock
In addition to a shared host response, differences were observed across sources. Comparison of the two most common sources (respiratory versus abdominal) demonstrated stronger host response anomalies in abdominal sepsis across all three pathophysiological domains. Of all groups, CNS infection was associated with the least host response aberrations. Markers of systemic inflammation were highest in abdominal and skin infections. Endothelial cell activation markers were largely similar across sources except for CNS (lower), whereas loss of vascular integrity, reflected by increased angiopoietin-2/1 ratio, was more profound in urinary, cardiovascular and skin infections. Stronger activation of the coagulation system was observed in abdominal and urinary infections, as indicated by lower levels of the natural anticoagulants protein $\mathrm{C}$ and antithrombin, and higher levels of D-dimer, as well as more prolongation of prothrombin time (PT) and activated partial thromboplastin time (aPTT). Similar results were obtained across sources after adjustment for confounders (group adjusted $p$ values shown below unadjusted $p$ values in Fig. 2; eTable 7, electronic supplementary material). 
Table 2 Pairwise comparison of 30-day mortality among sepsis patients with differences sources of infection

\begin{tabular}{|c|c|c|c|c|c|c|}
\hline Unadjusted & $\begin{array}{l}\text { Respiratory } \\
\text { OR }(95 \% \mathrm{Cl})\end{array}$ & $\begin{array}{l}\text { Abdominal } \\
\text { OR }(95 \% \mathrm{Cl})\end{array}$ & $\begin{array}{l}\text { Urinary } \\
\text { OR (95\% Cl) }\end{array}$ & $\begin{array}{l}\text { Cardiovascular } \\
\text { OR }(95 \% \text { Cl) }\end{array}$ & $\begin{array}{l}\text { CNS } \\
\text { OR }(95 \% \mathrm{Cl})\end{array}$ & $\begin{array}{l}\text { Skin } \\
\text { OR }(95 \% \mathrm{Cl})\end{array}$ \\
\hline Respiratory (ref) & na & $0.89(0.66-1.2)$ & $0.53(0.33-0.85)^{*}$ & $0.9(0.51-1.57)$ & $0.67(0.37-1.2)$ & $0.86(0.51-1.46)$ \\
\hline Abdominal (ref) & $1.12(0.83-1.52)$ & na & $0.6(0.36-0.99)^{*}$ & $1.01(0.56-1.84)$ & $0.75(0.4-1.41)$ & $0.97(0.55-1.71)$ \\
\hline Urinary (ref) & $1.88(1.17-3.02)^{*}$ & $1.67(1-2.81)^{*}$ & na & $1.69(0.84-3.41)$ & $1.26(0.61-2.6)$ & $1.62(0.82-3.19)$ \\
\hline Cardiovascular (ref) & $1.11(0.64-1.95)$ & $0.99(0.54-1.8)$ & $0.59(0.29-1.19)$ & na & $0.74(0.34-1.63)$ & $0.96(0.46-2.01)$ \\
\hline CNS (ref) & $1.5(0.83-2.70$ & $1.33(0.71-2.49)$ & $0.8(0.38-1.64)$ & $1.34(0.61-2.94)$ & na & $1.29(0.6-2.76)$ \\
\hline Skin (ref) & $1.16(0.68-1.97)$ & $1.03(0.58-1.83)$ & $0.62(0.31-1.22)$ & $1.04(0.5-2.19)$ & $0.78(0.36-1.67)$ & na \\
\hline Adjusted & OR $(95 \% \mathrm{Cl})$ & OR $(95 \% \mathrm{Cl})$ & OR $(95 \% \mathrm{Cl})$ & OR $(95 \% \mathrm{Cl})$ & OR $(95 \% \mathrm{Cl})$ & OR (95\% Cl) \\
\hline Respiratory (ref) & na & $0.68(0.47-0.98)^{*}$ & $0.46(0.27-0.78)^{*}$ & $0.95(0.51-1.77)$ & $1.12(0.59-2.14)$ & $0.81(0.44-1.49)$ \\
\hline Abdominal (ref) & $1.47(1.02-2.11)^{*}$ & na & $0.68(0.38-1.21)$ & $1.33(0.69-2.54)$ & $1.64(0.81-3.32)$ & $1.14(0.6-2.14)$ \\
\hline Urinary (ref) & $2.17(1.28-3.69)^{*}$ & $1.48(0.83-2.66)$ & na & $2.12(0.98-4.55)$ & $2.43(1.09-5.44)^{*}$ & $1.81(0.86-3.83)$ \\
\hline Cardiovascular (ref) & $1.05(0.56-1.96)$ & $0.75(0.39-1.44)$ & $0.47(0.22-1.02)$ & na & $1.22(0.52-2.85)$ & $0.86(0.38-1.93)$ \\
\hline CNS (ref) & $0.89(0.47-1.71)$ & $0.61(0.3-1.23)$ & $0.41(0.18-0.92)^{*}$ & $0.82(0.35-1.91)$ & na & $0.7(0.3-1.62)$ \\
\hline Skin (ref) & $1.23(0.67-2.25)$ & $0.88(0.47-1.66)$ & $0.55(0.26-1.17)$ & $1.17(0.52-2.64)$ & $1.43(0.62-3.30)$ & na \\
\hline
\end{tabular}

Only known single sources of infection shown (see eTable 5 for unknown, other and mixed sources). Model adjusted for age, sex, BMI, Charlson comorbidity score (without age), admission type, hospital site, causative pathogen, blood culture positive, the Acute Physiology Score, mSOFA score, and septic shock

$O R$ odds ratio, $\mathrm{Cl}$ confidence interval, ref reference category, na not applicable

${ }^{*} p<0.05$ versus the reference category

\section{Whole-blood leukocyte transcriptome analysis}

Further insight into the host response to infections from different sources was gained by studying the leukocyte blood transcriptome in a subgroup of sepsis patients enrolled during the first 1.5 years of the study period $(N=335,16.7 \%)$. This subgroup was comparable to the whole cohort (eTable 8, electronic supplementary material). First, we compared the transcriptome from each source of infection group against a group of healthy subjects $(N=42)$, and observed a strongly altered response for all sources (Figure S3, electronic supplementary material). Most differentially expressed genes (DEGs) were common among all sources; the total number of common DEGs was 4,126 (20.6\% of all genes measured) (Fig. 3A). Pathway enrichment analysis of these common DEGs revealed a number of significant pathways when compared to healthy which are consistent with earlier studies in sepsis patients (Fig. 3C) [18].

A large number of DEGs were unique to particular sources (Fig. 3A): 1188 DEGs were unique to cardiovascular, 1087 unique to CNS, 660 unique to skin and 182 unique to abdominal infections. Notably, there were no unique DEGs associated with pulmonary or urinary tract infections. Pairwise comparisons showed the largest overlap in the transcriptional response in abdominal and pulmonary infections (78\% of DEGs in common), whereas CNS and cardiovascular infections were most different (50\% of DEGs in common) (Fig. 3B). Next, we compared the transcriptional responses between the different sources of infection for targeted pathways using gene set enrichment analysis (Fig. 3D). We noted a heightened transcription of hemostasis pathways in abdominal and a dampened response in urosepsis, which was especially driven by the sub-pathway "platelet activation, signaling and aggregation". In contrast, when assessing the "cytokine signaling in the immune system" pathway, abdominal infections had a dampened response with prominent low interferon signaling. For "innate immune system" pathways, there was an overall heightened response in abdominal and skin infections and a dampened response in respiratory, urinary, and cardiovascular infections, which was driven by the sub-pathway "neutrophil degranulation". In addition, the "adaptive immune system" transcriptional response was heightened in abdominal, CNS, and skin infection compared to respiratory. Metabolism pathways were relatively downregulated in respiratory and upregulated in abdominal and skin infections, which was especially driven by differences in the sub-pathway "the TCA cycle and respiratory electron transport".

\section{Discussion}

The vast majority of sepsis cases originate from a specific anatomic source and part of the heterogeneity of sepsis has been attributed to differences in the primary site of infection. Nonetheless, sepsis trials that evaluated strategies manipulating the host response mostly did not take the primary site of infection into account. The primary objective of the current study was to determine to which 

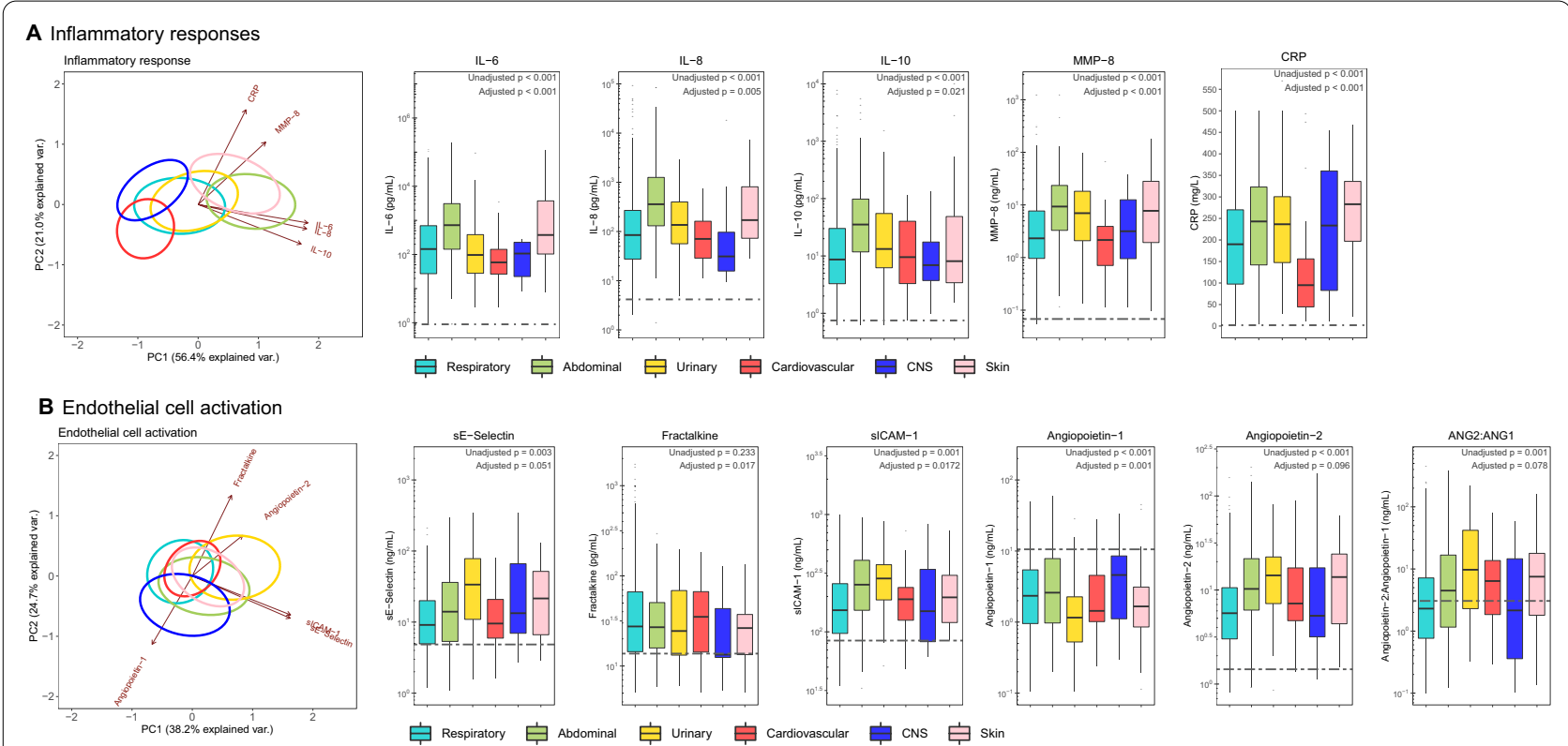

C Coagulation activation
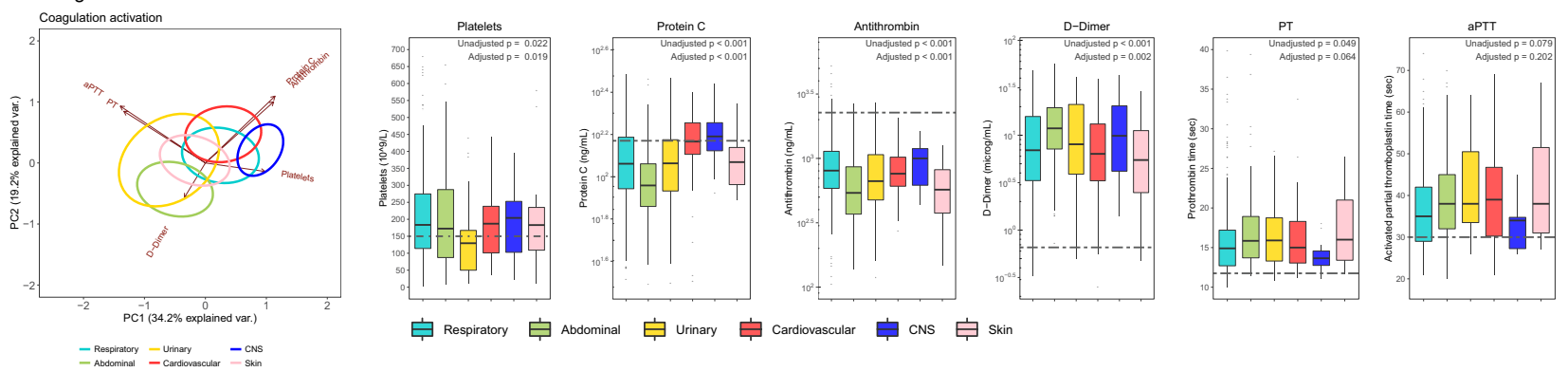

Fig. 2 Host response biomarkers in patients with sepsis on admission stratified according to the source of infection. Biological parameters are classified as $\mathbf{A}$ inflammatory responses, $\mathbf{B}$ endothelial cell activation, and $\mathbf{C}$ coagulation activation biomarkers. Data are presented as principal component analysis (PCA) plots (far left side of each row), and box and whiskers (with dotted lines in box plots representing median values obtained in 27 healthy age-matched healthy subjects). Ellipse circles of infection groups in PCA plots are drawn around patient data points (not shown here for clarity), wherein the centroid is the barycenter of the patient data points belonging to the same source of infection; arrows in PCA plots indicate direction of correlation of plasma markers with loadings of PCA components. $p$ values in box plots represent type-III Wald tests for the source of infection groups derived from linear regression models, wherein the adjusted model included age, sex, ethnicity, BMI, Charlson comorbidity score (without age), admission type, hospital site, blood culture positivity, type of causative pathogen, mSOFA score, Acute Physiology Score and shock. ANG angiopoietin, aPTT activated partial thromboplastin time, IL interleukin, MMP matrix metalloproteinase, PT prothrombin time, sE-Selectin soluble E-selectin, sICAM soluble intercellular adhesion molecule

extent systemic host response deviations differ between sepsis patients with distinct origins of infection. In this prospectively enrolled cohort of critically ill patients with sepsis, the anatomic source of infection was categorized by specified criteria and retrospective case-by-case review into 'pure' sources without coinfection or mixed infections with multiple sources [17]. By measuring 16 host response biomarkers providing insight into pathophysiological mechanisms implicated in sepsis and by analyzing transcriptomic profiles in blood leukocytes, we show that the specific source of infection triggers notable differences in host response aberrations in key pathways of the immune system.
Previous studies reported on the association between the source of infection and sepsis mortality $[5-8,10,16$, 23]. Consistent with our cohort, these investigations-if conducted on mixed ICUs-documented the respiratory tract as the most common infectious source, followed by the abdomen [5-7]. The source-specific mortality reported here is in agreement with earlier publications: several studies found a high mortality in sepsis patients with pneumonia, whereas sepsis originating from the urinary tract has been associated with a relatively low mortality $[5,6]$. An investigation entailing 7974 ICU patients showed that mortality due to abdominal sepsis varies between distinct diagnoses, with sepsis resulting from an 

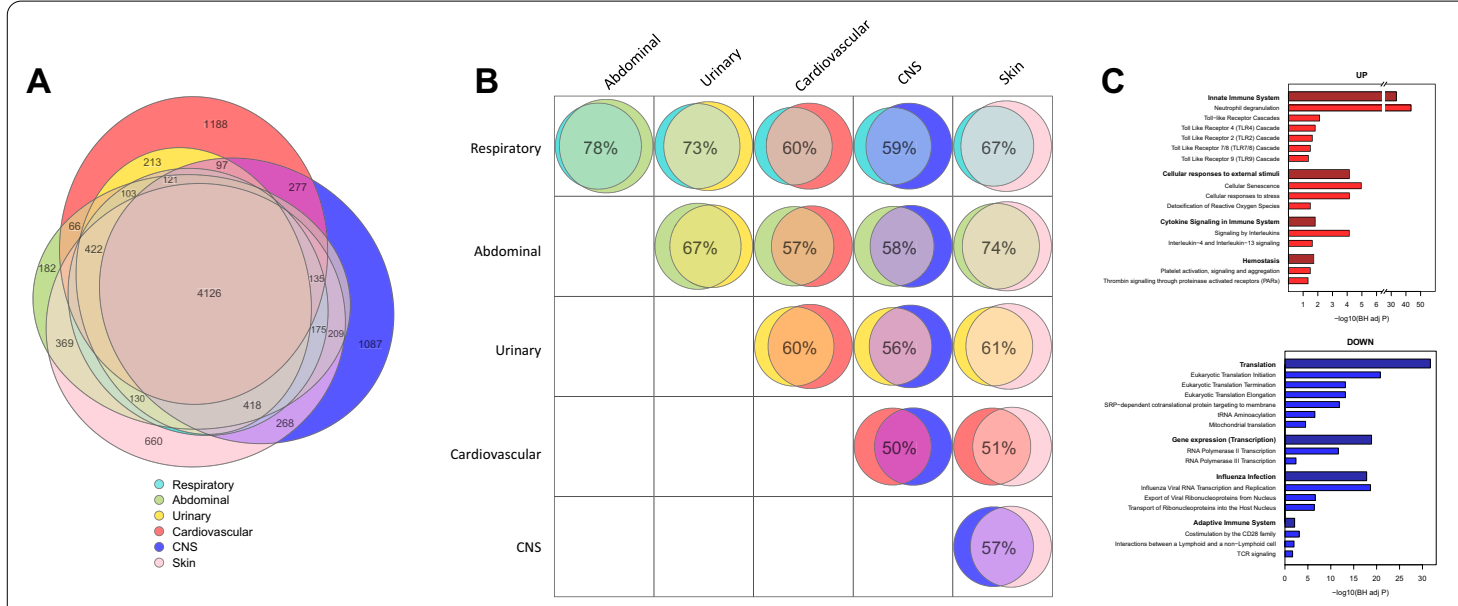

D
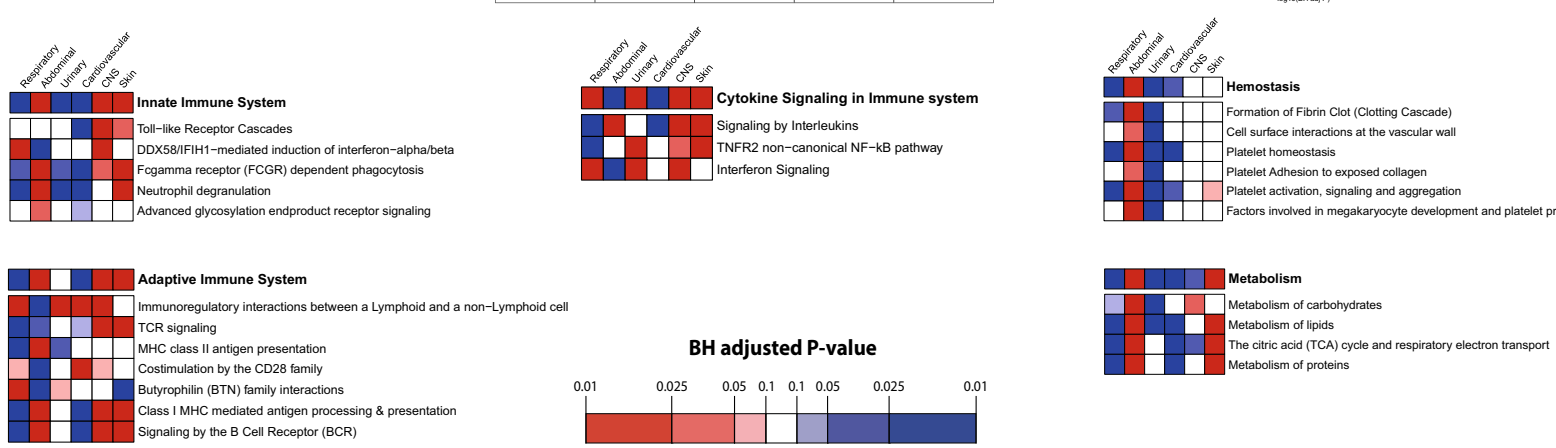

Fig. 3 Blood transcriptomics response in patients with sepsis on admission stratified according to the source of infection. A Venn-Euler diagram illustrating the shared and distinct leukocyte transcriptional responses between source of infection groups relative to health (with differential expressed genes according to high effect size $>0.8$ with Hedges $g$ ). Number of overlapping genes are shown if above 65 . B Pairwise comparison of source of infection groups showing common transcriptional response (with differential expressed genes according to medium effect size $>0.4$ with Hedges g). C Pathway analysis of the common response to sepsis (4126 genes common to all sources) relative to health. Canonical signaling subpathways were grouped into their parent pathway according to Reactome pathway database. D Comparing the blood transcriptional responses between the source of infection groups for targeted pathways. For every gene, expression values were scaled across all sepsis samples. Then, for each source of infection, the vector of mean expression values was ordered for gene set enrichment analysis implementing 1000 permutations. For each selected pathway, we summarize its enrichment by magnitude, using the $\mathrm{BH}$ adjusted $p$ value correcting for all existing Reactome database pathways, and direction using the normalized enrichment score (red $=+v e$, blue $=-v e$ )

ischemic bowel bearing the worst prognosis and sepsis secondary to cholangitis having a relatively low mortality [8]. Most patients with abdominal sepsis in our cohort suffered from peritonitis following bowel perforation; hospital mortality did not differ between patients with respiratory infection and those with a perforated viscus in the afore mentioned study [8], which is in line with our finding of similar unadjusted 30-day mortalities in patients with respiratory and abdominal sepsis. Out of the known sources, 30-day survival was worst in cardiovascular infection patients, in agreement with an earlier study [16]. In our cohort differences in mortality across infection sources remained after adjustment for confounding, corroborating earlier analyses showing independent associations between mortality and primary site of infection $[5,6,8]$.
Aberrations in key host response pathways implicated in sepsis pathogenesis differed across infectious sources, even after adjustments for factors that might impact the direction and/or extent of the host response, suggesting that the primary source at least in part drives the systemic reaction to infection. Hence, while the more pronounced proinflammatory response in abdominal and skin infections may have been related to the high proportion of shock in these groups, this does not fully explain host response differences between these sources. The circulating levels of the anti-inflammatory cytokine IL-10 were higher in abdominal sepsis than all other sources indicating a more disturbed immune response entailing both pro- and anti-inflammatory reactions. Endothelial cell activation and loss of vascular integrity were largely similar albeit prudently higher in urinary tract, skin and 
cardiovascular infections, with exception of CNS infection, which was less affected. Two endothelial cell activation markers, sE-selectin and sICAM-1, were highest in urinary tract infections, possibly due to high incidence of chronic renal insufficiency and diabetes in these patients [24]. Activation of the coagulation system was especially present in abdominal sepsis, as reflected by elevated D-dimer levels and reduced levels of the anticoagulant factors antithrombin and protein $\mathrm{C}$. This observation is in agreement with a previous study reporting that patients with abdominal infections were more likely to have early coagulation failure than patients with respiratory infections ( $17.3 \%$ vs. $9.5 \%$ respectively) [9]. Of interest, however, markers for so-called "sepsis-induced coagulopathy" (i.e., PT and platelet counts) [25] did not differ or only marginally differed between groups.

Knowledge of the association between blood leukocyte transcriptomes and source of infection in sepsis is limited and focused on differences between respiratory and abdominal sepsis $[15,26]$. In our cohort, besides finding a substantial common response, pairwise comparisons revealed unique source-specific DEGs. Abdominal and respiratory infections showed the largest overlap (76\% DEGs in common), which is in agreement with previous studies $[15,26]$. CNS and cardiovascular infections were the most different ( $47 \%$ in common). We demonstrated enrichment of gene expression related to interferon signaling in respiratory sepsis, which is in accordance with a study that compared patients with sepsis due to community-acquired pneumonia (CAP) or fecal peritonitis [15]. Enhanced interferon signaling may be driven by a preponderance of viral infections in respiratory sepsis [27]. Pathways reflecting immune suppression, including T-cell receptor (TCR) signaling and major histocompatibility complex (MHC) class I and II, were particularly downregulated in abdominal sepsis, which may have relevance for selection of patients for immune stimulatory therapies.

Our study has strengths and limitations. We studied a large prospectively enrolled cohort in which patients were characterized according to strict criteria. Data were collected several years ago (2011-2013) and guidelines for the management of sepsis (followed in both institutions) have changed in some aspects since then [28]. Therapeutic interventions and causative pathogens differ between sources, which may impact host response characteristics. Classification of sources into eight categories may be an oversimplification. We took low sample sizes in CNS and skin infections into account for analyzing the transcriptional host response using effect sizes instead of $p$ values. We robustly corrected the analyses for predisposed patient characteristics, infection-related information, and disease severity between the different sources of infection. Nonetheless, a bias may have remained due to unmeasured confounders. Data were collected in two ICUs in The Netherlands, which may limit the generalizability. Leukocyte transcriptomes were analyzed by micro-arrays rather than by RNA sequencing. Host response parameters were determined in patients with a high likelihood of infection only.

\section{Conclusions}

The heterogeneity of patients with sepsis makes stratification into subgroups with more similar pathobiological profiles a major challenge. We here show that after rigorous adjustments for confounders the source of infection remains an independent contributor to the extent and particularities of the host response. Our results provide further understanding of sepsis heterogeneity and suggest that the source of infection should be taken into account when selecting patients for trials testing immune modulatory drugs.

\section{Supplementary Information}

The online version contains supplementary material available at https://doi. org/10.1007/s00134-021-06574-0.

\section{Author details \\ ${ }^{1}$ Center for Experimental and Molecular Medicine, Amsterdam University Medical Centers, Location Academic Medical Center, University of Amster- dam, Room T1.240, Meibergdreef 9, 1105 AZ Amsterdam, The Netherlands. \\ ${ }^{2}$ The Amsterdam Institute for Infection and Immunity, Amsterdam University Medical Centers, Amsterdam, The Netherlands. ${ }^{3}$ Department of Intensive Care Medicine, Amsterdam University Medical Centers, Location Academic Medical Center, University of Amsterdam, Amsterdam, The Netherlands. \\ ${ }^{4}$ Laboratory of Experimental Intensive Care and Anesthesiology (L.E.I.C.A), Amsterdam University Medical Centers, Location Academic Medical Center, University of Amsterdam, Amsterdam, The Netherlands. ${ }^{5}$ Mahidol-Oxford Tropical Medicine Research Unit (MORU), Mahidol University, Bangkok, Thailand. ${ }^{6}$ Nuffield Department of Medicine, University of Oxford, Oxford, UK. ${ }^{7}$ Department of Medical Microbiology, University Medical Center Utrecht, Utrecht, The Netherlands. ${ }^{8}$ Julius Center for Health Sciences and Primary Care, University Medical Center Utrecht, Utrecht, The Netherlands. ${ }^{9}$ Department of Intensive Care Medicine, University Medical Center Utrecht, Utrecht, The Netherlands. ${ }^{10}$ Department of Clinical Epidemiology, Biostatistics and Bioin- formatics, Amsterdam UMC, Location Academic Medical Center, University of Amsterdam, Amsterdam, The Netherlands. ${ }^{11}$ Division of Infectious Diseases, Amsterdam University Medical Centers, Location Academic Medical Center, University of Amsterdam, Amsterdam, The Netherlands.}

\section{Acknowledgements}

This research was performed within the framework of the Center for Translational Molecular Medicine (CTMM) (www.ctmm.nl), project Molecular Diagnosis and Risk Stratification of Sepsis (Grant 04I-201). H.P.S. was supported by the Dutch Kidney Foundation (Kolff Grant Nr. 190K009). J.M.B. was supported by the European Commission (Horizon 2020, ImmunoSep, Grant number 847422). L.A.v.V. was supported by a VENI grant from ZonMW (Grant number 09150161910033). Members of MARS consortium are: Departments of Intensive Care Medicine, Amsterdam University Medical Centers, location Academic Medical Center, University of Amsterdam, Amsterdam, The Netherlands: Friso M. de Beer, MD, Lieuwe D. J. Bos, PhD, Gerie J. Glas, MD, Roosmarijn T. M. van Hooijdonk, MD, Janneke Horn, MD, Laura R. A. Schouten, MD, Marleen Straat, MD, Luuk Wieske, MD, Esther Witteveen, MD. Center for Experimental and Molecular Medicine, Amsterdam University Medical Centers, location Academic Medical Center, University of Amsterdam, Amsterdam, The Netherlands, and Center of Infection and Immunity Amsterdam (CINIMA), Amsterdam University Medical Centers, location Academic Medical Center, University of Amsterdam, Amsterdam, The Netherlands; Tom D.Y. Reijnders, 
MD, Alex R. Schuurman, MD, Tjitske S.R. van Engelen, MD, Liza Pereverzeva, MD, Arie J. Hoogendijk, PhD, Mischa A. Huson, MD, Maryse A. Wiewel, MD; Department of Medical Microbiology, Department of Intensive Care Medicine, and Julius Center for Health Sciences and Primary Care, University Medical Center Utrecht, Utrecht, the Netherlands; Peter M.C. Klein Klouwenberg, MD, David S.Y. Ong, MD; Department of Intensive Care Medicine and Julius Center for Health Sciences and Primary Care, University Medical Center Utrecht, Utrecht, the Netherlands; Jos F. Frencken, MD, Maria E. Koster-Brouwer, MSc, Kirsten van de Groep, MD, Diana M. Verboom, MD.

\section{Declarations}

\section{Funding}

This research was performed within the framework of the Center for Translational Molecular Medicine (CTMM) (www.ctmm.nl), project Molecular Diagnosis and Risk Stratification of Sepsis (Grant 04l-201). The sponsor CTMM was neither involved in the design and conduction of the study nor was the sponsor involved in collection, management, analysis, and interpretation of the data or preparation, review or approval of the article. Decision to submit the article was not dependent on the sponsor.

\section{Conflicts of interest}

The authors disclose that they do not have any potential conflicts of interest.

\section{Ethics approval}

Patients were included via an opt-out consent method approved by the institutional review boards of both hospitals (IRB No. 10-056C).

\section{Consent to participate}

Patients were included via an opt-out consent method approved by the institutional review boards of both hospitals (IRB No. 10-056C).

\section{Consent to publish}

Not applicable/all data used for the present study have been anonymized and the submission does not include information that may identify individual persons.

\section{Availability of data, code and materials}

Gene expression datasets are available at the Gene Expression Omnibus public repository of NCBI under accession number GSE65682. Other data generated and/or analyzed during the current study are available on reasonable request. All requests should be sent to t.vanderpoll@amsterdamumc.nl.

\section{Author contributions}

HPS, JMB, FU, BPS, LAvV, and TvdP contributed to the study design. HPS, JMB, FU, BPS, LAvV, and TvdP accessed and verified the data. HPS, JMB, and TvdP drafted the manuscript. HPS and JMB performed all the data analyses. All the authors contributed to interpretation of the data, critically revised the content, and gave final approval for publication.

\section{Open Access}

This article is licensed under a Creative Commons Attribution-NonCommercial 4.0 International License, which permits any non-commercial use, sharing, adaptation, distribution and reproduction in any medium or format, as long as you give appropriate credit to the original author(s) and the source, provide a link to the Creative Commons licence, and indicate if changes were made. The images or other third party material in this article are included in the article's Creative Commons licence, unless indicated otherwise in a credit line to the material. If material is not included in the article's Creative Commons licence and your intended use is not permitted by statutory regulation or exceeds the permitted use, you will need to obtain permission directly from the copyright holder. To view a copy of this licence, visit http://creativecommons.org/licen ses/by-nc/4.0/.

\section{Publisher's Note}

Springer Nature remains neutral with regard to jurisdictional claims in published maps and institutional affiliations.

\section{References}

1. Singer M, Deutschman CS, Seymour CW, Shankar-Hari M, Annane D, Bauer $\mathrm{M}$ et al (2016) The Third international consensus definitions for sepsis and septic shock (Sepsis-3). JAMA 315(8):801-810. https://doi.org/ 10.1001/jama.2016.0287

2. Rudd KE, Johnson SC, Agesa KM, Shackelford KA, Tsoi D, Kievlan DR et al (2020) Global, regional, and national sepsis incidence and mortality, 1990-2017: analysis for the global burden of disease study. Lancet 395(10219):200-211. https://doi.org/10.1016/S0140-6736(19)32989-7

3. Angus DC, van der Poll T (2013) Severe sepsis and septic shock. N Engl J Med 369(21):2063. https://doi.org/10.1056/NEJMc1312359

4. Stanski NL, Wong HR (2020) Prognostic and predictive enrichment in sepsis. Nat Rev Nephrol 16(1):20-31. https://doi.org/10.1038/ s41581-019-0199-3

5. Abe T, Ogura H, Kushimoto S, Shiraishi A, Sugiyama T, Deshpande GA et al (2019) Variations in infection sites and mortality rates among patients in intensive care units with severe sepsis and septic shock in Japan. J Intensive Care 7:28. https://doi.org/10.1186/s40560-019-0383-3

6. Jeganathan N, Yau S, Ahuja N, Otu D, Stein B, Fogg L et al (2017) The characteristics and impact of source of infection on sepsis-related ICU outcomes. J Crit Care 41:170-176. https://doi.org/10.1016/j.jcrc.2017.05. 019

7. Klastrup V, Hvass AM, Mackenhauer J, Fuursted K, Schonheyder HC, Kirkegaard $\mathrm{H}$ et al (2016) Site of infection and mortality in patients with severe sepsis or septic shock. A cohort study of patients admitted to a Danish general intensive care unit. Infect Dis (Lond). 48(10):726-731. https://doi.org/10.3109/23744235.2016.1168938

8. Leligdowicz A, Dodek PM, Norena M, Wong H, Kumar A, Kumar A et al (2014) Association between source of infection and hospital mortality in patients who have septic shock. Am J Respir Crit Care Med 189(10):12041213. https://doi.org/10.1164/rccm.201310-18750C

9. Volakli E, Spies C, Michalopoulos A, Groeneveld AB, Sakr Y, Vincent JL (2010) Infections of respiratory or abdominal origin in ICU patients: what are the differences? Crit Care 14(2):R32. https://doi.org/10.1186/cc8909

10. Zahar JR, Timsit JF, Garrouste-Orgeas M, Francais A, Vesin A, DescorpsDeclere A et al (2011) Outcomes in severe sepsis and patients with septic shock: pathogen species and infection sites are not associated with mortality. Crit Care Med 39(8):1886-1895. https://doi.org/10.1097/CCM. 0b013e31821b827c

11. Sinapidis D, Kosmas V, Vittoros V, Koutelidakis IM, Pantazi A, Stefos A et al (2018) Progression into sepsis: an individualized process varying by the interaction of comorbidities with the underlying infection. BMC Infect Dis 18(1):242. https://doi.org/10.1186/s12879-018-3156-z

12. Gogos C, Kotsaki A, Pelekanou A, Giannikopoulos G, Vaki I, Maravitsa $P$ et al (2010) Early alterations of the innate and adaptive immune statuses in sepsis according to the type of underlying infection. Crit Care 14(3):R96. https://doi.org/10.1186/cc9031

13. Pelekanou A, Tsangaris I, Kotsaki A, Karagianni V, Giamarellou H, Armaganidis A et al (2009) Decrease of CD4-lymphocytes and apoptosis of CD14-monocytes are characteristic alterations in sepsis caused by ventilator-associated pneumonia: results from an observational study. Crit Care 13(6):R172. https://doi.org/10.1186/cc8148

14. Poukoulidou T, Spyridaki A, Mihailidou I, Kopterides P, Pistiki A, Alexiou Z et al (2011) TREM-1 expression on neutrophils and monocytes of septic patients: relation to the underlying infection and the implicated pathogen. BMC Infect Dis 11:309. https://doi.org/10.1186/1471-2334-11-309

15. Burnham KL, Davenport EE, Radhakrishnan J, Humburg P, Gordon AC, Hutton P et al (2017) Shared and distinct aspects of the sepsis transcriptomic response to fecal peritonitis and pneumonia. Am J Respir Crit Care Med 196(3):328-339. https://doi.org/10.1164/rccm.201608-16850C

16. Stortz JA, Cox MC, Hawkins RB, Ghita GL, Brumback BA, Mohr AM et al (2020) Phenotypic heterogeneity by site of infection in surgical sepsis: a prospective longitudinal study. Crit Care 24(1):203. https://doi.org/10. 1186/s13054-020-02917-3

17. Klein Klouwenberg PM, Ong DS, Bos LD, de Beer FM, van Hooijdonk RT, Huson MA et al (2013) Interobserver agreement of centers for disease control and prevention criteria for classifying infections in critically ill patients. Crit Care Med 41(10):2373-2378. https://doi.org/10.1097/CCM. Ob013e3182923712 
18. van Vught LA, Klein Klouwenberg PM, Spitoni C, Scicluna BP, Wiewel MA, Horn J et al (2016) Incidence, risk factors, and attributable mortality of secondary infections in the intensive care unit after admission for sepsis. JAMA 315(14):1469-1479. https://doi.org/10.1001/jama.2016.2691

19. Scicluna BP, van Vught $L A$, Zwinderman $A H$, Wiewel MA, Davenport $E E_{\text {, }}$ Burnham KL et al (2017) Classification of patients with sepsis according to blood genomic endotype: a prospective cohort study. Lancet Respir Med 5(10):816-826. https://doi.org/10.1016/S2213-2600(17)30294-1

20. Klein Klouwenberg PM, Cremer OL, van Vught LA, Ong DS, Frencken JF, Schultz MJ et al (2015) Likelihood of infection in patients with presumed sepsis at the time of intensive care unit admission: a cohort study. Crit Care 19:319. https://doi.org/10.1186/s13054-015-1035-1

21. Cranendonk DR, van Vught LA, Wiewel MA, Cremer OL, Horn J, Bonten MJ et al (2017) Clinical characteristics and outcomes of patients with cellulitis requiring intensive care. JAMA Dermatol 153(6):578-582. https://doi.org/ 10.1001/jamadermatol.2017.0159

22. Pierrakos C, Velissaris D, Bisdorff M, Marshall JC, Vincent JL (2020) Biomarkers of sepsis: time for a reappraisal. Crit Care 24(1):287. https://doi.org/10. 1186/s13054-020-02993-5

23. Motzkus CA, Luckmann R (2017) Does infection site matter? A systematic review of infection site mortality in sepsis. J Intensive Care Med 32(8):473-479. https://doi.org/10.1177/0885066615627778

24. Zonneveld R, Martinelli R, Shapiro NI, Kuijpers TW, Plotz FB, Carman CV (2014) Soluble adhesion molecules as markers for sepsis and the potential pathophysiological discrepancy in neonates, children and adults. Crit Care 18(2):204. https://doi.org/10.1186/cc13733

25. Iba T, Nisio MD, Levy JH, Kitamura N, Thachil J (2017) New criteria for sepsis-induced coagulopathy (SIC) following the revised sepsis definition: a retrospective analysis of a nationwide survey. BMJ Open 7(9):e017046. https://doi.org/10.1136/bmjopen-2017-017046

26. Zador Z, Landry A, Balas M, Marshall JC, Cusimano MD (2020) Data driven analysis reveals shared transcriptome response, immune cell composition, and distinct mortality rates across differing etiologies of critical illness. Crit Care Med 48(3):338-343. https://doi.org/10.1097/CCM.00000 00000004128

27. Jain S, Self WH, Wunderink RG, Fakhran S, Balk R, Bramley AM et al (2015) Community-acquired pneumonia requiring hospitalization among US Adults. N Engl J Med 373(5):415-427. https://doi.org/10.1056/NEJMo a1500245

28. Rhodes A, Evans LE, Alhazzani W, Levy MM, Antonelli M, Ferrer R et al (2017) Surviving sepsis campaign: international guidelines for management of sepsis and septic shock: 2016. Intensive Care Med 43(3):304-377. https://doi.org/10.1007/s00134-017-4683-6 